\title{
The Influence of Magnetic History on the Stability of Critical State and the Dynamics of Flux Jumps in Conventional NbTi Superconductor
}

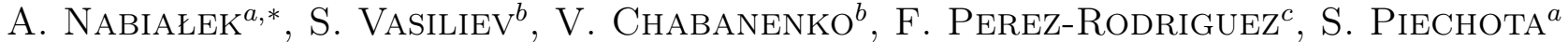 \\ AND H. SZYMCZAK ${ }^{a}$ \\ ${ }^{a}$ Institute of Physics, Polish Academy of Sciences, al. Lotników 32/46, 02-668 Warsaw, Poland \\ ${ }^{b}$ Institute for Physics and Engineering, NASU, 72 ul. R. Luxemburg, 83114 Donetsk, Ukraine \\ ${ }^{c}$ Instituto de Fisica, Benemerita Universidad Autonoma de Puebla, Apdo. Post. J-48 Puebla, 72570 Pue., Mexico
}

\begin{abstract}
We studied the influence of magnetic history on the stability of critical state and the dynamics of flux jumps in conventional NbTi superconductor. We have found that both the stability of critical state and the dynamics of flux jumps are strongly influenced by the magnetic history and by the magnetic field distribution induced within the sample by preceding jumps. This magnetic field distribution can be particularly complex in the region of the hysteresis loop where the external magnetic field changes its sign (region of remagnetization), because of demagnetizing effects. For this reason, the most complex structure of the flux jumps is observed in the third quadrant of magnetization hysteresis loop. Flux jumps observed in the region of remagnetization have different dynamics in comparison to the jumps observed in other regions of the magnetization hysteresis loop. Duration of these jumps is usually significantly longer than others, and their structure is more complex.
\end{abstract}

PACS numbers: 74.70.Ad, 74.25.Sv

\section{Introduction}

Thermomagnetic avalanches, called flux jumps, are commonly observed in hard type II superconductors [1]. These phenomena cause problems in practical applications of superconductors. Superconductors used in applications are usually characterized by high critical current densities and, as a result, by strong magnetic hysteresis. Hence, it is necessary to understand deeper the influence of the magnetic history on critical state stability and on the dynamics of flux jumps in these materials. It is well known that the stability of the critical state depends on the magnetic field distribution in the superconducting sample, and that this distribution is determined by the magnetic history. This problem was already analyzed by Sosnowski [2], Müller and Andrikidis [3] and also in our previous works $[4,5]$. The influence of the magnetic history on the dynamics of the flux jumps remains poorly understood.

Here we study experimentally the influence of the magnetic history on critical state stability and on the dynamics of flux jumps in conventional NbTi superconductor. We discuss the influence of demagnetizing effects on the structure of the flux jumps. In particular, we focus on the flux jump structure in the region of remagnetization.

* corresponding author; e-mail: nabia@ifpan.edu.pl

\section{Experiment}

In our studies, we used a $4.5 \times 4.5 \times 4.5 \mathrm{~mm}^{3}$ cube of conventional NbTi 60 at.\% superconductor. In order to increase the critical current density, the sample was annealed for $50 \mathrm{~h}$ at $420^{\circ} \mathrm{C}$. Magnetization measurements were performed using a conventional VSM magnetometer with maximal field attainable of $16 \mathrm{kOe}$. Additionally, a pick-up coil (7 turns of copper wire) was wound up around the investigated sample and connected to data acquisition board in the computer. The sample with the coil was put into the cryostat of VSM. Such system enabled us simultaneous registration of the magnetic moment of the sample and of the dynamic changes of the magnetic flux in the sample. The pick-up coil was used to study dynamics of the flux jumps.

\section{Results}

Figure 1 shows a magnetization hysteresis loop of our sample taken at $4.2 \mathrm{~K}$. Before taking the hysteresis loop, the sample was cooled in zero magnetic field (see the virgin, ZFC, curve in Fig. 1). One can see five pronounced jumps of the magnetic moment caused by the thermomagnetic avalanches. In Fig. 1, there is one jump (J1) in the first quadrant (q1) of the magnetization hysteresis loop, one jump (J2) in the second quadrant (q2), two jumps (J3, J4) in the third quadrant (q3), and one jump (J5) in the fourth quadrant (q4). For further discussion, 
it is important to notice that the jump in the second quadrant (J2) is complete, so the magnetic moment drops to zero during this jump (see point p3 in Fig. 1), and this jump occurs in the external magnetic field which is very close to zero.

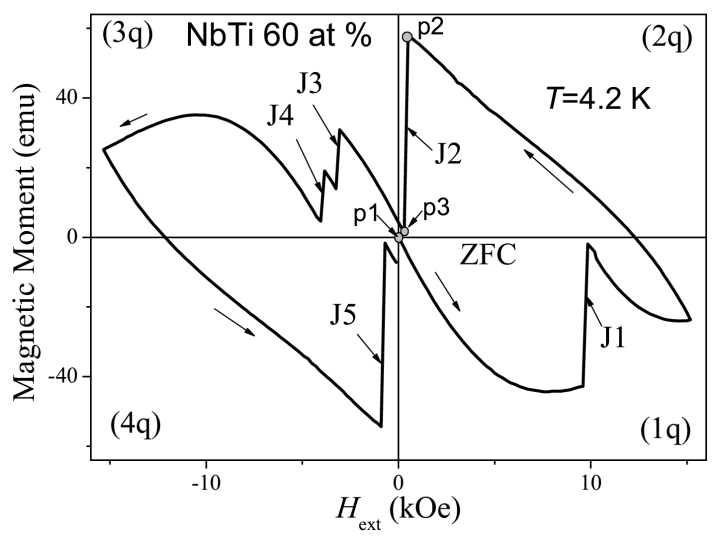

Fig. 1. Magnetization hysteresis loop of the $4.5 \times 4.5 \times$ $4.5 \mathrm{~mm}^{3} \mathrm{NbTi}$ cube taken at $4.2 \mathrm{~K}$. One can see five jumps (J1-J5) of the magnetic moment caused by thermomagnetic avalanches.

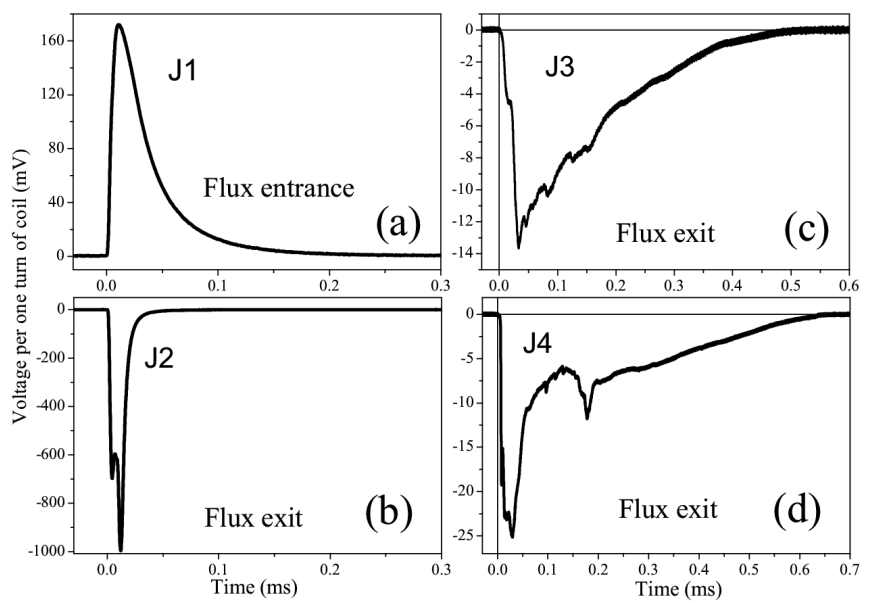

Fig. 2. Temporal changes of the voltage per one turn of the coil during four jumps observed in our experiment. (a) The jump in the first quadrant, (b) the jump in the second quadrant, (c) and (d) two following jumps in the third quadrant.

Figure 2 presents the dynamics of the flux jumps studied in our experiment. Figures $2 \mathrm{a}-\mathrm{d}$ show temporal changes of the voltage on the coil wound up around the investigated sample during the following jumps. Figure 1a presents the dynamics of the first flux jump. The structure of this jump is simple; we observe a single peak in voltage. The structure of the following jumps (Figs. 1b-d) is complicated. Figure 1b shows the dynamics of the jump in the second quadrant. Figure 1c and 1d show dynamics of the two jumps in the third quadrant. The most complex structure belongs to the jumps in the third quadrant. The duration of these jumps is one or two orders of magnitude longer and the amplitude one or two orders of magnitude smaller than of other jumps observed in the experiment.

\section{Discussion}

Let us consider the magnetic field distribution in our sample after second flux jump (see J2 in Fig. 1). The magnetic moment after this jump is zero and the external magnetic field is close to zero (point p3 in Fig. 1). Hence, we could expect the magnetic field distribution in this point of the hysteresis loop to be the same as after cooling the sample in zero magnetic field (point p1 - starting point of the hysteresis loop), and as a result the structure of the flux jumps in the third quadrant to be the same as in the first quadrant. Surprisingly, the structure of the flux jumps in the third quadrant is very different than in the first quadrant. We can explain this fact as follows.

Although, after the second flux jump, the magnetic moment of the sample is equal to zero, the magnetic field distribution is NOT the same as after cooling the sample in zero magnetic field. In our opinion, after the second flux jump, the local magnetic field induction in the sample is not uniformly distributed within the sample volume, but we have some regions of the sample with positive local magnetization coexisting with the regions of negative local magnetization. Such complex magnetic field distribution may also, after integration over the sample volume, give the magnetic moment of the whole sample equal to zero.

This hypothesis is supported by the results of our investigation of local magnetic induction at the surface of a superconducting NbTi cylinder during magnetic avalanches [6]. Our experiment was performed with the aid of a miniature Hall probe. It was found that after a thermomagnetic avalanche local surface magnetization changes its sign.

If the magnetic field induction is not uniformly distributed in the sample volume after the second flux jump, the conditions of the flux jumps occurrence in the third quadrant of magnetization hysteresis loop are different than in the first quadrant. As a result, we can also expect different dynamics of the flux jumps in the third quadrant. The coexistence of the regions with positive and negative local magnetization means that during a flux jump, a process of annihilations of vortex-antivortex pairs may occur. The Meissner holes and turbulent structures [7] can play an important role in avalanche magnetic flux dynamics as well.

Let us now consider, how such complex magnetic field distribution after the second flux jump may occur. To understand this, it is necessary to take into account demagnetizing effects. Our sample has cubic form. From the initial part of the virgin magnetization curve, we have estimated the demagnetizing factor $D$ to be about 0.36 . For a qualitative discussion, let us take the formula for internal magnetic field in the sample 


$$
H_{\text {int }}=H_{\text {ext }}-D M,
$$

where $H_{\text {ext }}$ is the external magnetic field and $M$ is the magnetization of the sample.

Just before the second flux jump (see point p2 in Fig. 1), the magnetic moment of the investigated sample was about $58 \mathrm{emu}$ (see Fig. 1), which gives an average magnetization $\mu_{0} M \approx 0.8 \mathrm{~T}$ after dividing by the sample volume. In this case, according to Eq. (1), the internal magnetic field before the second flux jump is negative, $\mu_{0} H_{\text {int }} \approx-0.3 \mathrm{~T}$. During the second flux jump, the average magnetization of the sample decreases to zero, and thus, according to Eq. (1), we can expect an increase (a decrease of a negative value) of the internal magnetic field, $H_{\text {int }}$.

On the other hand, during a flux jump, the temperature of the sample increases and the density of the superconducting screening current is reduced. In the sample with zero demagnetizing factor and for descending branch of the magnetization hysteresis loop, this process leads always to a decrease of the local magnetic field induction in the superconducting sample. It is important to note that Eq. (1) can be applied only if the sample magnetization is constant in the whole sample volume, and in the case of a hard type II superconductors, it can be used only as a rough approximation. Nevertheless, in the case of a superconducting sample with nonzero demagnetizing factor, we can expect a coexistence of two opposite processes during a flux jump: (1) a decrease of the superconducting screening currents, (2) an increase of internal magnetic field caused by demagnetizing effects. In the region of remagnetization, these two opposite processes induce screening currents with opposite directions, and after the flux jump, we can obtain a complex magnetic field distribution with the coexistence of the regions with positive and negative local magnetization.

\section{Conclusions}

Demagnetizing effects strongly influence the structure and the dynamics of the flux jumps in the region of remagnetization. After a flux jump, we can obtain a complex magnetic field distribution in this region, with coexisting regions of positive and negative local magnetization. Such complex magnetic field distribution determines the structure and dynamics of the succeeding flux jumps.

\section{Acknowledgments}

This work was partly supported by Polish Ministry of Science and Higher Education under research project for years 2007-2009 (grant N N202 1285 33).

\section{References}

[1] R.G. Mints, A.L. Rakhmanov, Rev. Mod. Phys. 53, 551 (1981).

[2] J. Sosnowski, J. Appl. Phys. 59, 4190 (1986).

[3] K.H. Müller, C. Andrikidis, Phys. Rev. B 49, 1294 (1994).

[4] V.V. Chabanenko, A.I. D'yachenko, A.V. Chabanenko, M.V. Zalutsky, H. Szymczak, S. Piechota, A. Nabiałek, Supercond. Sci. Technol. 11, 1181 (1998).

[5] V.V. Chabanenko, A.I. D'yachenko, M.V. Zalutskii, V.F. Rusakov, H. Szymczak, S. Piechota, A. Nabiałek, J. Appl. Phys. 88, 5875 (2000).

[6] V.V. Chabanenko, S. Vasiliev, A. Nabiałek, V. Rusakov, P. Aleksyeyev, H. Szymczak, S. Piechota, Physica C 460-462, 776 (2007).

[7] V.K. Vlasko-Vlasov, U. Welp, G.W. Grabtree, D. Gunter, V.V. Kabanov, V.I. Nikitenko, L.M. Paulius, Phys. Rev. B 58, 3446 (1998). 